\title{
Análisis de componentes de material frágil por medio de métodos numéricos ${ }^{(\bullet)}$
}

\author{
M.D. Rubio ${ }^{(*)}$, C.J. Crespo $^{(*)}$, R. Jakel $^{(*)}$, P. Dietz $^{(*)}$, A. Villellas $^{(* *)}$ y M. Doblaré(***)
}

Resumen Los materiales frágiles deben sus características al elevado número de microgrietas producidas en su conformación. Es por ello por lo que han ido apareciendo una serie de criterios de cálculo específicos para estos materiales que se basan en cálculos de tipo probabilista y en las propiedades del material definidas por Weibull. En el I.M.W. (Clausthal, Alemania) se ha desarrollado un programa de cálculo de resistencia de piezas de estos materiales, por ejemplo cerámicas, que trabaja como subprocesador de un programa de elementos finitos, MARC, de forma que permite analizar la probabilidad de vida de las piezas según tres criterios generales y criterios de mecánica de fractura (once para elementos de volumen y seis para elementos de superficie). Asimismo, el programa permite calcular la probabilidad de fallo, según estos criterios en el caso de que se realicen cálculos con carga aplicada durante intervalos de tiempo, y realizar cálculos de factores de seguridad con los que se ha diseñado la pieza.

Palabras clave: Cerámicas. Probabilidad de fallo. Criterios de fractura. Elementos finitos.

\section{Use of numerical methods to calculate the reliability of components fabricated with fragile materials}

\begin{abstract}
Some materials are fragile due to the great number of microcraks produced during their conformation processes, for example ceramics. That is the reason why, along these years different design criteria have been proposed to calculate these fragile materials, specially ceramics, based in probabilistic theories. A programme to calculate ceramic components with these criteria has been developed in the I.M.W. (Clausthal, Germany). This program works as a subprocessor of the finite element program MARC, and can analyse the probability of survival of a component with three general criteria and with fracture mechanics criteria (eleven when calculating volume components and six for surface ones). Those criteria have been implemented also to calculate the probability of survival with applied loads during time intervals and to calculate security factors.
\end{abstract}

Keywords: Ceramics. Probability of failure. Fracture criteria. Finite elements.

\section{INTRODUCCIÓN}

Actualmente, se constata que los materiales que presentan un acentuado carácter frágil no cumplen las teorías de fallo desarrolladas para los materiales dúctiles convencionales. Como consecuencia, ha aparecido una serie de teorías que proponen un

(•) Trabajo recibido el día 16 de junio de 1997.

(*) Institut für Maschinenwesen (T.U. Clausthal). Robert Koch Str. 38678 Clausthal-Zellerfeld (Alemania).

(**) Dpto. de Ciencia de Materiales e Ingeniería Metalúrgica. Centro Politécnico Superior. Universidad de Zaragoza. C/ María de Luna, 3. 50015-Zaragoza (España).

(***) Dpto. de Mecánica de Medios Continuos y Teoría de Estructuras. Centro Politécnico Superior. Universidad de Zaragoza. diseño para estos materiales basado en el carácter marcadamente probabilista que presenta la supervivencia de componentes realizados en materiales frágiles ante diferentes estados de carga.

El uso de estas teorías probabilistas conlleva una elevada complejidad de cálculo lo que implica la necesidad del cálculo informático para el análisis de piezas de este material.

En este artículo se presenta un programa para este tipo de cálculos, WEIBULL2, desarrollado como subrutina de postproceso del programa de elementos finitos MARC, ya que WEIBULL2 utiliza para sus cálculos el estado tensional de cada punto de la pieza que provee MARC. Es de resaltar que WEIBULL2 puede utilizarse con mínimos cambios como postprocesador de cualquier otro 
programa universal de elementos finitos (por ejemplo, también ha sido implementada una primera versión en ABAQUS) con la única condición de permitir acceder a su base de datos.

A partir de este estado de tensiones, el programa es capaz de calcular en cada punto de la pieza la tensión resultante según diferentes criterios, la probabilidad de fallo en ese punto y la probabilidad de fallo total de la pieza, no sólo para cargas instantáneas sino también para cargas aplicadas durante intervalos de tiempo que, además, pueden ser variables.

Con todo esto, se pretende dotar al ingeniero de una herramienta de cálculo de componentes frágiles que le permita obtener resultados estadísticos de probabilidad de supervivencia global de piezas de geometría arbitraria, sometidas a estados de carga multiaxiales, pudiendo estas variar con el tiempo. Además de ello, es posible obtener también mapas de probabilidad de fallo a lo largo de toda la pieza, con lo cual se obtiene una idea clara de los puntos más críticos en el diseño.

\section{CARACTERÍSTICAS DE LOS MATERIALES CERÁMICOS}

Existe una elevada gama de materiales frágiles tales como algunos composites y aleaciones, pero los materiales cerámicos (incluyendo el cristàl estructural) pueden considerarse como uno de los más claros ejemplos de material con comportamiento frágil.

En los últimos años, en el campo industrial, debido a las solicitaciones cada vez más extremas a las que se someten los materiales empleados, se han buscado diferentes materiales que pudiesen presentar unos rangos de uso que no podían ser cubiertos por los metales tradicionales de una forma óptima. Los materiales cerámicos presentan, en la actualidad, una serie de propiedades específicas que potencian su interés tecnológico: elevada dureza, resistencia a altas temperaturas y a ambientes corrosivos y abrasivos, mucho mayor que la que presentan en la actualidad metales de elevadas prestaciones, así como una densidad mucho más baja que estos, lo que permite un ahorro de costes energéticos al reducir el peso de sus componentes en comparación con los metálicos.

Sin embargo, existe un grave inconveniente que ha frenado hasta el momento una aplicación generalizada de estos materiales cerámicos: su elevada fragilidad. El causante de esta característica es el sistema de conformación de piezas de este material. La conformación a partir de polvos cerámicos produce la aparición de una distribución de grietas microscópicas y defectos a lo largo del volumen de la pieza. Asimismo, los procesos de tratamiento superficial inducen la formación de una serie de grietas en la superficie, lo que permite asegurar la existencia de una distribución de defectos en ésta completamente independiente de la que caracteriza al volumen del componente. Es este conjunto de defectos el que produce un efecto de intensificación sobre el campo de tensiones que sufre el material. El crecimiento catastrófico de alguna de estas grietas es el causante de la rotura de la pieza y, por tanto, del comportamiento frágil de los materiales cerámicos y el que debe de justificar el criterio teórico empleado para el análisis.

$\mathrm{El}$ análisis de materiales frágiles hace necesario considerar una serie de factores que no son precisos en el estudio de los materiales dúctiles. Así como en los segundos es suficiente analizar la resistencia del material en aquellos puntos en los que el estado de tensiones es más crítico, cuando se trabaja con materiales frágiles se pueden tener zonas de la pieza más críticas ante un estado de tensiones determinado que otras donde, sin embargo, las tensiones sean mayores. Esto se debe a que aunque en un punto el estado de tensiones es mayor que en otros, se puede producir el fallo del material en otro punto en el que la distribución de defectos es más crítica, fallando ante tensiones menores de las que resiste el punto más solicitado. Por ello, es importante tener en cuenta en el análisis todo el volumen de la pieza, así como emplear un punto de vista probabilístico para su estudio, de forma que todas las partes de la pieza contribuyen a la probabilidad de supervivencia del conjunto. Además, será conveniente suponer una distribución de grietas a lo largo de la pieza cuyo tamaño y orientación se han de tratar también desde el punto de vista probabilista y no constante. Es muy importante aquí destacar la utilización del concepto de "la unión o eslabón más débil" que es el que más se ajusta a los resultados experimentales, de forma que según él, la pieza falla en el instante en que alguna de sus grietas rompe, al igual que una cadena rompe al hacerlo uno de sus eslabones.

\section{TEORÍAS PROBABILISTAS PARA EL ANÁLISIS DE MATERIALES FRÁGILES}

\subsection{Criterios generales o fenomenológicos}

Weibull (1) fue el primero que presentó un tratamiento probabilista para este tipo de materiales frágiles, buscando explicar la dispersión de valores observada en diferentes ensayos. Aplicando sus resultados a una serie de ensayos realizados con probetas sometidas a carga de flexión o tracción, pero extrapolando los resultados a ensayos teóricos de tipo uniaxial, se planteó unas curvas tensión-probabilidad de fallo que, en escala doble logarítmica 
en probabilidades, se ajustaban a líneas rectas (Fig. 1 ); esto le permitió plantear una función de probabilidad de tipo exponencial definiendo unas propiedades características del material.

El valor de probabilidad de fractura de una probeta de volumen, $\mathrm{V}$, es, por tanto:

$$
P_{\mathrm{f}}=1-\exp \left[\frac{V}{V_{0}}\left(\frac{\sigma-\sigma_{\mathrm{u}}}{\sigma_{0}}\right)^{\mathrm{m}}\right]
$$

Que expresado en dobles logaritmos de la probabilidad de fallo permitían encontrar sobre la curva de forma directa los parámetros característicos del material:

$$
\ln \ln \left[\frac{1}{1-P_{\mathrm{fV}}}\right]=m_{\mathrm{v}} \ln \left(\sigma-\sigma_{\mathrm{uV}}\right)+\ln \frac{V}{V_{0}}-m_{\mathrm{v}} \ln \sigma_{0 \mathrm{~V}}
$$

Esta expresión fue desarrollada por Weibull para carga uniaxial, considerando que el material sólo podía fallar sometido a estados de tracción.

Fue él mismo quien planteó la posibilidad de extrapolar los resultados para carga multiaxial, evaluando una tensión normal media en cada subvolumen de la pieza y aplicándole un tratamiento análogo al anterior. Este método, intuitivamente lógico, carecía de base teórica suficiente como para poder ser aceptado como generalización para cualquier estado de carga.
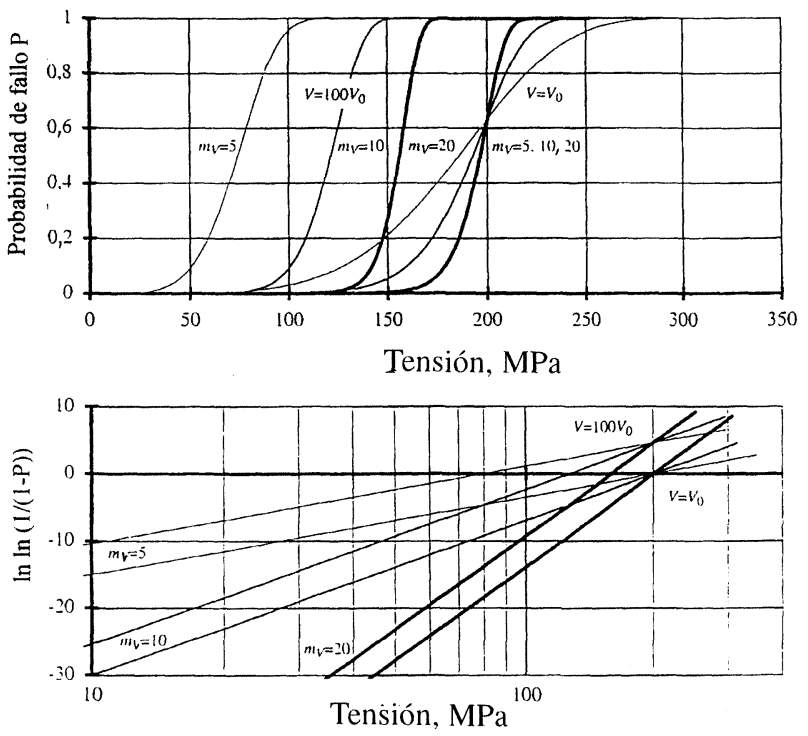

FIG. 1.- Curvas de probabilidad de fallo para diferentes materiales cerámicos, y su representación en escala doble logarítmica.

FIG. 1.- Probability of failure-curves for different ceramic materials and its representation in double logarithms.
Tampoco tienen justificación teórica los métodos presentados posteriormente por:

Barnett (2) - Freudenthal (3). Según la combinación de sus teorías, el estado de tensiones multiaxial produce un efecto análogo en el riesgo de fractura a considerar estas tensiones como actuando de forma independiente (Principio de acciones independientes), admitiendo también la suposición incorrecta de que las tensiones de compresión no influyen en la fractura de la grieta.

$$
\sigma_{\mathrm{VeqPIA}}=\sqrt[m_{v}]{\sigma_{1}^{m_{v}}+\sigma_{2}^{m_{v}}+\sigma_{3}^{m_{v}}} \operatorname{con} \sigma_{i}>0
$$

Weibull - Stanley (4): Teoría desarrollada como síntesis de ambas fuentes, busca un factor de equivalencia de efectos entre las aportaciones de tensiones de tracción y compresión actuantes en un punto de la pieza $\left(H\left(\sigma_{\mathrm{i}}\right)\right)$, de forma que pueda reflejar la influencia de la compresión en la tensión resultante transformándola en tensión de tracción equivalente.

$$
\begin{gathered}
P_{\text {fvws }}(\sigma)=1-\exp \left\{-\frac{1}{V_{0} \sigma_{0 v}} \int_{v}\left[\left(\frac{\sigma_{1}-\sigma_{u}}{H\left(\sigma_{1}\right)}\right)^{\mathrm{m}_{\mathrm{v}}}+\right.\right. \\
\left.\left.+\left(\frac{\sigma_{2}-\sigma_{\mathrm{u}}}{H\left(\sigma_{2}\right)}\right)^{\mathrm{m}_{\mathrm{v}}}+\left(\frac{\sigma_{3}-\sigma_{\mathrm{u}}}{H\left(\sigma_{3}\right)}\right)^{\mathrm{m}_{\mathrm{v}}}\right]^{\mathrm{d} V}\right\}
\end{gathered}
$$

Beierlein (5): considera que las tensiones que actúan sobre la pieza aumentando su riesgo de fractura son producidas por las deformaciones principales positivas; su hipótesis ha sido ampliamente utilizada en la construcción de componentes refractarios con mucho éxito.

$P_{\mathrm{fVBe}}=1-\exp \left\{-\frac{1}{V_{0} \cdot \sigma_{0 \mathrm{~V}}^{\mathrm{mV}}} \int_{V}\left(\sigma_{1 B e}^{m_{\mathrm{v}}}+\sigma_{2 B e}^{m_{\mathrm{v}}}+\sigma_{3 B e}^{m_{\mathrm{v}}}\right) \mathrm{d} V\right.$

con:

$$
\sigma_{\mathrm{iBe}}=\sigma_{\mathrm{i}}-\mathrm{v} \cdot\left(\sigma_{\mathrm{j}}-\sigma_{\mathrm{k}}\right) \geq 0
$$

Englobados todos dentro de lo que se denominan métodos fenomenológicos, buscan describir el comportamiento del material, desarrollando variaciones de la expresión de Weibull para explicar comportamientos a compresión y ante estados de carga multiaxial, sin presentar una justificación teórica a este comportamiento, ya que obvian reflejar la razón física del comportamiento frágil, las grietas. Esto conlleva una omisión de la influencia de los cortantes, tan importantes al justificar la fractura. 


\subsection{Criterios de mecánica de fractura}

Son las teorías de mecánica de fractura las que proporcionan una base adecuada para el estudio de la probabilidad de fallo en materiales frágiles.

Según estas teorías, la probabilidad de supervivencia de un componente no es más que el producto de las probabilidades de supervivencia de todos los diferenciales de volumen de los que está compuesto, de forma análoga al planteamiento inicial de Weibull:

$$
P_{\mathrm{sV}}=\left(1-\Delta P_{\mathrm{fVI}}\right)^{\mathrm{n}} \cong \prod_{1}^{\mathrm{n}} e^{-\Delta \mathrm{P}_{\mathrm{fvi}}}=e-\sum^{\mathrm{n} \Delta \mathrm{P}_{\mathrm{fvi}}}=e^{-\mathrm{R}_{\mathrm{f}}}
$$

Es por tanto la distribución de grietas en el material, tanto las embebidas en el volumen como las situadas en su superficie, la causante tanto del comportamiento frágil como de la dispersión del valor de resistencia a la fractura.

Así, se puede definir en cada diferencial del volumen (o de superficie) una función de densidad de grietas $N\left(\sigma_{\mathrm{cr}}\right)$ determinada por la tensión crítica a la que estas fallan $\left(\sigma_{\mathrm{cr}}\right)$.

Esto permite definir el riesgo de fractura en cada $\mathrm{d} V$ o d $S$ para un estado de tensiones dado, (que se denotará como $\Sigma$ ) como el producto de dos términos de probabilidad;

$$
\Delta P_{\mathrm{f}}\left(\Sigma, \sigma_{\mathrm{cr}}, \mathrm{d} V\right)=\Delta P_{\mathrm{f} 1} \cdot P_{\mathrm{f} 2}
$$

Si se trabaja en un $\Delta V$, el valor de $\Delta P_{\mathrm{f} 1} \cdot P_{\mathrm{f} 2}$ es;

$$
\Delta P_{\mathrm{f} 1}=\Delta V \frac{\mathrm{d} N\left(\sigma_{\mathrm{cr}}\right)}{\mathrm{d} \sigma_{\mathrm{cr}}} \mathrm{d} \sigma_{\mathrm{cr}}
$$

es decir, este término presenta la probabilidad de que en este $\Delta V($ o $\Delta S)$ se encuentre una grieta de tensión crítica comprendida entre $\sigma_{\mathrm{cr}}$ y $\sigma_{\mathrm{cr}}+\mathrm{d} \sigma_{\mathrm{cr}}$.

Debido a que la resistencia de las grietas está directamente relacionada con la longitud de éstas, este término da una medida implícita de las dimensiones de la grieta, de forma que no será necesario que aparezca el valor de longitud de grieta de forma explícita, aunque según el tipo de grieta que se considere predominante, el factor de forma tendrá un valor diferente.

El segundo término se define como la probabilidad de que una grieta se encuentre orientada en este $\Delta V$ o $\Delta S$, de forma que el estado de tensiones $(\Sigma)$ aplicado en el diferencial esté produciendo una $\sigma_{\mathrm{eq}}$ $\geq \sigma_{\text {cr }}$ en la orientación en la que se encuentra la grieta. Se definirá para un $\Delta V$ como:

$$
P_{\mathrm{f} 2}=\frac{\Omega\left(\Sigma, \sigma_{\mathrm{cr}}\right)}{4_{\pi}}
$$

Apareciendo en la expresión el área del ángulo sólido como área en la que $\sigma_{\mathrm{eq}} \geq \sigma_{\mathrm{cr}}$ dividida por todo el área de la esfera de radio unidad $(4 \pi)$.

Así pues, la expresión de la $P_{\mathrm{f}}$ de un $\mathrm{d} V_{\mathrm{i}}$ de material cerámico es:

$$
P_{\mathrm{fi}}=1-e^{-\Delta V_{\mathrm{i}}} \cdot 1-e^{-\Delta V_{\mathrm{i}}} \cdot \int_{0}^{\sigma_{\mathrm{emix}}} \frac{\Omega\left(\Sigma, \sigma_{\mathrm{cr}}\right)}{4 \pi} \cdot \frac{\mathrm{d} N_{\mathrm{v}}\left(\sigma_{\mathrm{cr}}\right)}{\mathrm{d} \sigma_{\mathrm{cr}}} \mathrm{d} \sigma_{\mathrm{cr}}
$$

Tal y como se ha planteado hasta el momento en las teorías de mecánica de la fractura, en cada $\mathrm{d} V$ (todo el razonamiento desarrollado es análogo para $\mathrm{d} S$ ) será necesario realizar una integral que depende del estado de tensiones al que está sometido. Por ello, el cálculo de la probabilidad de fractura asociada a cada punto de integración es realmente complicado. Aún así, Nemeth (6) presentó un programa de elementos finitos, llamado CARES, que realizaba este tipo de integrales.

Fue Thiemier (7) quien propuso un método para cambiar los límites de esta integral $\left(0-\sigma_{\text {eqmáx }}\right)$ de forma que la probabilidad de fallo de un volumen dado eș:

$$
P_{\mathrm{f}}=1-e^{-} \int_{\mathrm{V}}\left[\int_{0}^{\pi} \int_{0}^{2 \pi} N\left(\sigma_{\mathrm{eq}}\right) \operatorname{sen} \alpha \cdot \mathrm{d} \beta \cdot \mathrm{d} \alpha\right] \mathrm{dV}
$$

mientras que para calcular la probabilidad de fallo de una superficie, se utilizará la expresión:

$$
P_{\mathrm{f}}=1-e^{-} \int_{\mathrm{S}}\left[\int_{0}^{2 \pi} N\left(\sigma_{\mathrm{eq}}\right) \mathrm{d} \alpha\right] \mathrm{dS}
$$

Las expresiones de $N\left(\sigma_{\text {eq }}\right)$ que las diferentes teorías de mecánica de fractura han ido planteando, tanto para criterios de volumen como de superficie son del tipo:

$$
N\left(\sigma_{\mathrm{cr}}\right)=K_{\mathrm{eq}} \cdot \sigma_{\mathrm{cr}}^{\mathrm{m}}
$$

siendo $K_{\text {eq }}$, para todas las teorías existentes, el factor de equivalencia para estados de carga uniaxial respecto a los resultados presentados por Weibull, aceptados como correctos para este estado de carga y que dependen de propiedades $\left(m, \sigma_{0}\right)$ que caracterizan el comportamiento del material.

Las diferentes teorías de mecánica de la fractura implementadas en el programa WEIBULL2 son las que se presentan a continuación pudiendo escoger el usuario un criterio diferente para los cálculos de superficie y los de volumen.

\subsubsection{Criterio de Griffith de tensiones máximas (Batdorf (8)) para cálculos en volumen:}

\section{Grieta Griffith:}




$$
\sigma_{\mathrm{eq}}=\frac{1}{2}\left(\sigma_{\mathrm{n}}+\sqrt{\sigma_{\mathrm{n}}^{2}+\tau_{\mathrm{eff}}^{2}}\right)
$$

Grieta Penny Shaped:

$$
\sigma_{\mathrm{eq}}=\frac{1}{2}\left(\sigma_{\mathrm{n}}+\sqrt{\sigma_{\mathrm{n}}^{2}+\left(\frac{2}{2-v} \tau_{\mathrm{eff}}\right)^{2}}\right)
$$

\subsubsection{Criterio de insensibilidad al cortante,} desarrollado a partir de las teorías de Thiemier (7) para cálculos en volumen

Grieta Griffit:

$$
\sigma_{\mathrm{eq}}=\frac{1}{2}\left(\alpha \cdot \tau_{\mathrm{eff}}+\sqrt{4 \sigma_{\mathrm{n}}^{2}+\left(\alpha \tau_{\mathrm{eff}}\right)^{2}}\right)
$$

Grieta Penny Shaped:

$$
\sigma_{\mathrm{eq}}=\frac{1}{2}\left(\left(\frac{2 \alpha}{2-v}\right) \tau_{\mathrm{eff}}+\sqrt{4 \sigma_{\mathrm{n}}^{2}+\left(\frac{2 \alpha}{2-v} \tau_{\mathrm{eff}}\right)^{2}}\right)
$$

\subsubsection{Criterio de la velocidad de energía coplanar de deformación (Batdorf (8))}

Grieta Griffith para volumen y superficie:

$$
\sigma_{\mathrm{eq}}=\sqrt{\sigma_{\mathrm{n}}^{2}+\tau_{\mathrm{eff}}^{2}}
$$

Grieta Penny Shaped, sólo para cálculo de volumen:

$$
\sigma_{\mathrm{eq}}=\sqrt{\sigma_{\mathrm{n}}^{2}+\left(\frac{2 \tau_{\mathrm{eff}}}{2-v}\right)^{2}}
$$

Grietas tipo Griffith Notch sólo para superficie:

$$
\sigma_{\text {eq }}=\sqrt{\sigma_{\mathrm{n}}^{2}+\left(\frac{0,7951}{1-v}\right)} \tau_{\text {eff }}^{2}
$$

\subsubsection{Criterio de Shetty (9)}

$$
\sigma_{\mathrm{eq}}=\frac{1}{2}\left(\sigma_{\mathrm{n}}+\sqrt{\sigma_{\mathrm{n}}^{2}+\left(2 \alpha \tau_{\mathrm{eff}}\right)^{2}}\right)
$$

Grietas Griffith para volumen y superficie:
Grietas Penny Shaped, para volumen:

$$
\sigma_{\text {eq }}=\frac{1}{2}\left(\sigma_{n}+\sqrt{\sigma_{n}^{2}+\left(\frac{4 \alpha}{2-v} \tau_{\text {eff }}\right)^{2}}\right)
$$

Grieta de tipo Griffith Notch para superficie:

$$
\sigma_{\text {eq }}=\frac{1}{2}\left(\sigma_{\mathrm{n}}+\sqrt{\sigma_{\mathrm{n}}^{2}+3,1803\left(\alpha \tau_{\mathrm{eff}}\right)^{2}}\right)
$$

Grieta semicirculares para superficie:

$$
\sigma_{\text {eq }}=\frac{1}{2}\left(\sigma_{\mathrm{n}}+\sqrt{\sigma_{\mathrm{n}}^{2}+3,301\left(\alpha \tau_{\mathrm{eff}}\right)^{2}}\right)
$$

\subsubsection{Criterio empírico desarrollado a partir de las teorías de Thiemier (7) para cálculos en volumen}

Grieta Griffith:

$$
\sigma_{\text {eq }}=\sqrt[u]{\sigma_{\mathrm{n}}^{\mathrm{u}}+\left(\alpha \tau_{\mathrm{eff}}\right)^{\mathrm{u}}}
$$

Grieta Penny Shaped:

$$
\sigma_{\mathrm{eq}}=\sqrt[u]{\sigma_{\mathrm{n}}^{u}+\left(\frac{2 \alpha}{2-v} \tau_{\mathrm{eff}}\right)^{u}}
$$

Para todos los casos el valor de $\sigma_{\mathrm{n}}$ ha de ser positivo, ya que en caso contrario será tomado como nulo, mientras que el valor de $\tau_{\text {eff }}$ dependerá a su vez del de la $\sigma_{\mathrm{n}}$ de forma que será:

$\mathrm{Si}$

$$
\begin{array}{lll}
\sigma_{\mathrm{n}} \geq 0 & \rightarrow & \tau_{\text {eff }}=\tau \\
\sigma_{\mathrm{n}}<0 & \rightarrow & \begin{array}{ll}
\tau_{\text {eff }}=\tau+\mu \cdot \sigma_{\mathrm{n}} & \text { si } \tau_{\text {eff }} \geq 0 \\
\tau_{\text {eff }}=0 & \text { si } \tau_{\text {eff }} \geq 0
\end{array}
\end{array}
$$

Siendo $\mu$ la fricción interna entre las caras de la grieta (Alpa (10)).

\section{PROGRAMA WEIBULL2}

Según se ha expuesto, el cálculo de componentes cerámicos conlleva la necesidad de utilizar programas de cálculo informático, por la gran cantidad de cálculos necesarios de forma diferencial en toda la pieza sin que se pueda obviar el tener en cuenta el total del volumen.

Por ello, se planteó el uso de programas de elementos finitos para poder, partiendo de los cálculos de tensiones que estos proporcionan, aplicar estas teorías en cada punto de integración, y así poder calcular probabilidades de fallo. 
El Institut für Maschinenwesen (IMW) utilizó el programa de elementos finitos MARC ya que ofrecía la flexibilidad suficiente para permitir al usuario acceder en cada punto de integración a los resultados ya calculados por él, tales como las tensiones, y para que el usuario disponiendo de ellos, pudiese realizar diversos cálculos, que el programa almacenaba como variables de cada punto, siendo posible después extrapolarlos a los nodos de forma automática. De esta forma, se desarrolló inicialmente un conjunto de subrutinas de MARC, empleadas para el cálculo probabilístico de materiales identificados como cerámicos, y se le llamó WEIBULL, en honor a este autor, permitiendo realizar cálculos de probabilidad según los tres primeros criterios generales presentados en este artículo (Jakel (11 y 12)). Posteriormente, se amplió la capacidad del programa, aumentándose las posibilidades de cálculo con los criterios de mecánica de fractura, tanto para volumen como para superficie. A esta nueva versión del programa se le llamó WEIBULL2.

El programa MARC ofrece al usuario una serie de subrutinas con las que él pueda realizar aquellos cálculos que le interesen. En el programa WEIBULL2 se emplean dos de estas, PLOTV y USDATA para calcular en cada punto de integración las variables escogidas por el usuario (Fig. 2). Además de ello, para realizar la lectura de datos de los distintos materiales cerámicos y por ello específicos para este tipo de cálculos, el usuario tendrá que situar dichos datos, en el fichero de lectura de datos, *.dat.

Plantear cálculos de mecánica de fractura obligó a realizar no sólo una evaluación de la tensión equivalente en cada punto de integración, sino también realizar en cada uno integrales de orientación, en la esfera de radio unidad, para cálculos en volumen y en la circunferencia de radio unidad para cálculos en superficie.

Además, se detectó un fallo de MARC en el cálculo del volumen y tensiones principales para casos de geometría cilíndrica, que también fue subsanado con este programa.

\section{RESULTADOS Y APLICACIONES}

Partiendo de un análisis de elementos finitos, el programa WEIBULLL2 puede calcular para cada punto de integración de la pieza el estado de tensiones principales al que está sometido, así como el volumen o superficie asociado a él, según el tipo de elemento desde el que se esté llamando al programa y las coordenadas de sus nodos. Puede reconocer si el elemento es de tipo volumétrico o superficial y si el material asociado al elemento es de carácter frágil o no, si lo es, calculará la tensión resultante según los tres criterios generales vistos, y según

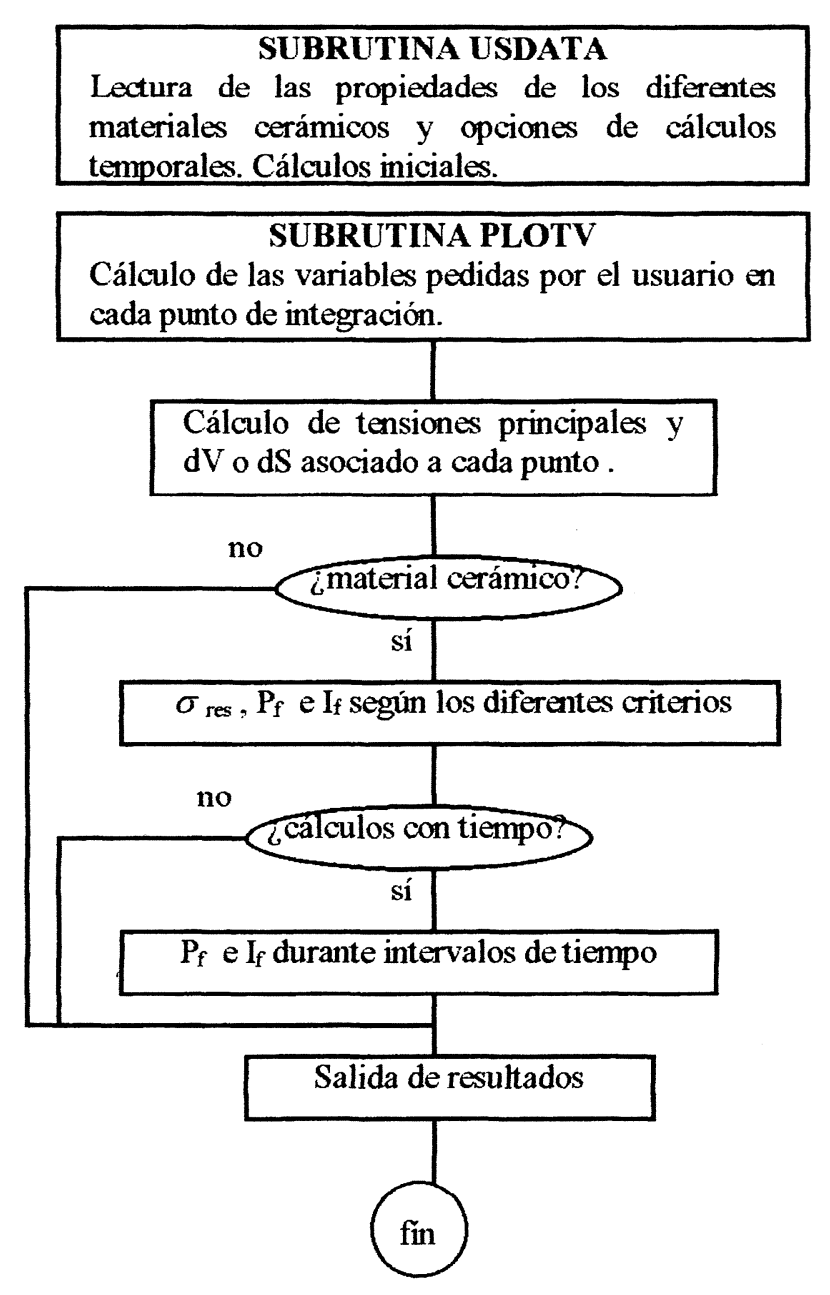

FIG. 2.- Algoritmo del programa WEIBULL2.

FIG. 2.- WEIBULL2's algorithm.

uno de los criterios de mecánica de fractura escogido por el usuario (que puede ser diferente dependiendo de que el elemento sea de volumen o superficie).

Calculada la tensión resultante en cada punto, según cada criterio, se calcula la $P_{\mathrm{f}}$ de carácter instantáneo asociada a ese punto, así como la $P_{\mathrm{f}}$ cuando la carga se aplica durante un intervalo de tiempo, por medio de la expresión desarrollada por Nadler (13) y cuando esta carga varía durante diferentes intervalos de tiempo, presentándose mapas de tensiones resultantes, probabilidades de fallo e intensidades de fallo para todos los tipos de cargas. Se diferenciará cuando los cálculos se realicen para elementos de volumen o para superficie, ya que los criterios aplicados son diferentes y las propiedades del material pueden variar.

Es importante localizar no sólo las zonas de mayor probabilidad de fallo en la pieza sino la probabilidad de fallo total de la pieza. Esto se hace a través de las expresiones: 


$$
P_{\mathrm{f}}=1-\exp \left\{-\frac{1}{V_{0} \sigma_{0}^{\mathrm{m}}} \sum_{\mathrm{i}=1}^{\mathrm{n}}\left[\sum_{\mathrm{j}=1}^{\mathrm{k}} V_{\mathrm{ij}}\left(\sigma_{\text {res }}-\sigma_{\mathrm{u}}\right)^{\mathrm{m}}\right]\right\}
$$

para cálculos estáticos, y por

$$
P_{\mathrm{f}}=1-\exp \left\{-\frac{1}{V_{0} \sigma_{0}^{\mathrm{m}}} \sum_{\mathrm{i}=1}^{\mathrm{n}}\left[\sum_{\mathrm{j}=1}^{\mathrm{k}} V_{\mathrm{ij}}\left[\sum_{\mathrm{l}=1}^{\mathrm{m}}\left(\sigma_{\mathrm{ijl}}-\sigma_{\mathrm{u}}\right)^{\mathrm{m} / \mu_{\mathrm{ul}}}\right]^{\mu}\right]\right\}
$$

para cargas aplicadas durante intervalos de tiempo (Fig. 3).

También se calcula, para una probabilidad de fallo límite dada por el usuario, el valor del factor de seguridad resultante con el que se ha diseñado el componente.

Con estos resultados, el usuario puede no sólo analizar su pieza sino también aplicar métodos automáticos de disminución de esta probabilidad según Crespo (14) y, por ello, de optimización de la pieza.

\subsection{Ventiladores cerámicos. Ejemplo de aplicación}

El desarrollo de este programa se ha aplicado al cálculo por elementos finitos de diversas piezas de material cerámico, entre ellas cabe destacar el diseño de un ventilador cerámico ( $\mathrm{SiSiC})$ de $55 \mathrm{~cm}$ de diámetro para unas condiciones de funcionamiento de $1300{ }^{\circ} \mathrm{C}$ a $2.400 \mathrm{rpm}$.

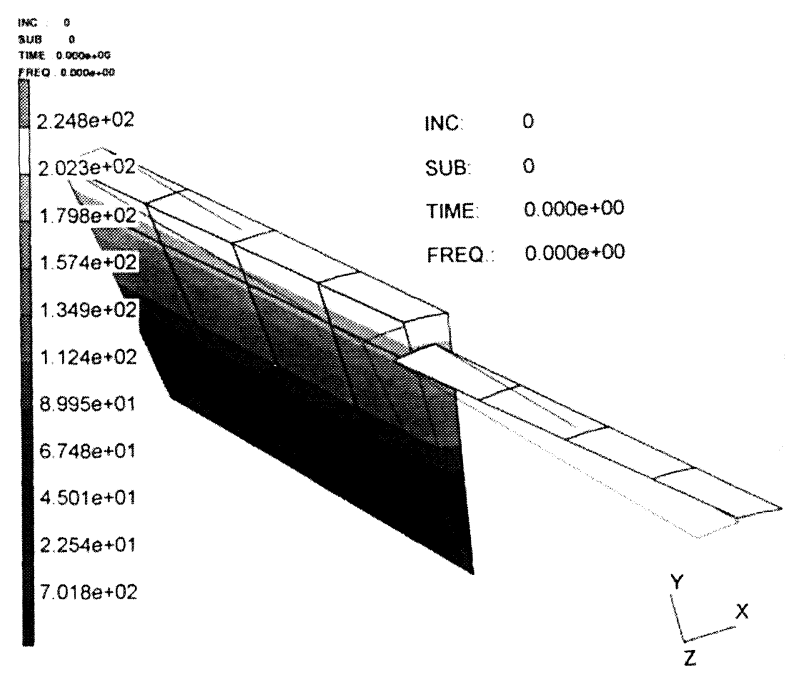

FIG. 3.- Cálculo de probabilidad de fallo de un cilindro a torsión según un criterio de mecánica de fractura.

FIG. 3.- Cylinder probability of failure when suffering torsion according to a fracture mechanic criterion.
Se analizaron por medio del programa de elementos finitos diferentes condiciones de funcionamiento (funcionamiento estacionario, enfriamiento repentino y proceso de calentamiento), de forma que se realizó un análisis probabilístico de todos ellos. La conclusión de los casos analizados fue que el estado de tensiones más crítico era el de enfriamiento repentino a $1000{ }^{\circ} \mathrm{C}$, y que la probabilidad de fallo total de la pieza se encontraba en torno a $0,03 \%$ (variando según los diferentes criterios alrededor de este valor) (Fig. 4). En la actualidad este ventilador se encuentra funcionando en perfectas condiciones, pudiéndose asegurar que su análisis por medio de este programa fue decisivo en su diseño.

\section{DISCUSIÓN Y CONCLUSIONES}

El diseño de materiales frágiles, tales como cerámicas, conlleva la aplicación de metodologías de diseño diferentes a las empleadas con materiales dúctiles, que reflejen los diversos aspectos inherentes a estos materiales, tales como la distribución aleatoria de grietas en su interior, de tamaño también aleatorio, lo que obliga a la consideración de todo el volumen de la pieza en el cálculo de su probabilidad de fractura, y no sólo de las zonas de tensión máxima.

La necesidad de tener en cuenta la totalidad del volumen y su estado tensional en el cálculo de probabilidades, hace muy importante el uso de programas de elementos finitos que permitan acceder a esta información en diferenciales de volumen o superficie, lo suficientemente pequeños como para

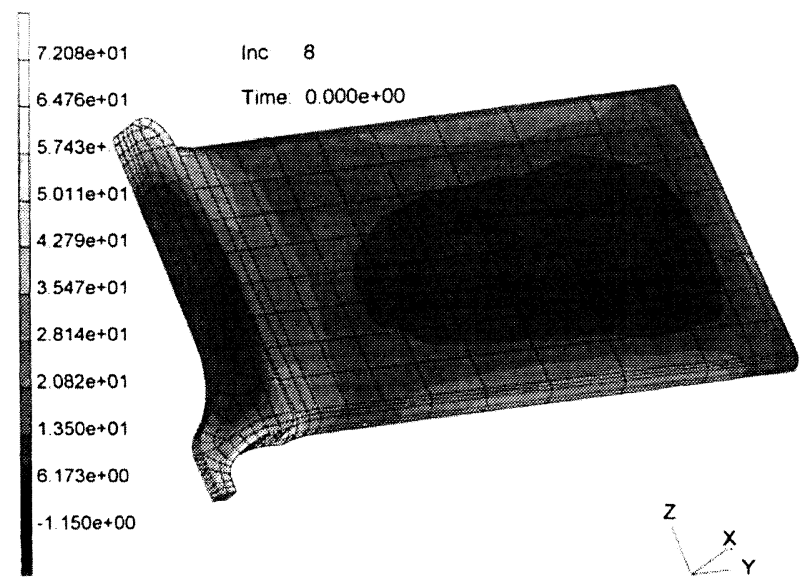

Fig. 4.- Tensiones resultantes de un álabe de un ventilador sufriendo un proceso de enfriamiento de 1.300 a $1.000{ }^{\circ} \mathrm{C}$ según un criterio de mecánica de fractura.

FIG. 4.- Fan blade resultant tension when suffering shock cooling from 1300 to $1000{ }^{\circ} \mathrm{C}$ according to a fracture mechanic criterion. 
que se pueda considerar en ellos un estado tensional constante.

Además, la aplicación de las diversas teorías, permite comparar los resultados probabilísticos obtenidos con criterios generales, con los criterios de mecánica de fractura (con una base teórica mucho más sólida). Ante estados de tracción, las diferencias entre ellos apenas son cuantitativas, mientras que en estados de tracción-compresión, las diferencias son más notables reflejando claramente la influencia de las tensiones de cortadura sobre la tensión resultante según cada criterio, y para estados de compresión, las diferencias entre ambos tipos de criterios todavía son mayores.

Hasta el momento, los resultados experimentales no permiten destacar alguno de los criterios de mecánica de fractura como el más apropiado, sino que según sea el tipo de estado tensional, los resultados se acercan más a uno u otro criterio. Aunque los criterios de carácter fenomenológico divergen de los de mecánica de la fractura de forma cuantitativa, su simplicidad hace que su uso sea interesante.

\section{REFERENCIAS}

(1) Weibull, W. A Statistical Theory of the Strength of Materials. Proceedings of the Royal Swedish Institute of Engineering Research. No. 151, 1939: 5-41.

(2) BARNETT, R.L. et al. Fracture of brittle materials under transient mechanical and thermal loading; AFFDL-TR66-220. 1967.

(3) Freudenthal, A.M. Statistical approach to brittle facture. Fracture Vol. 2, Liebowitz; Academic Press, Nueva York, 1968: 591-619.
(4) Fessler, H., Sivill, A.Dl y Stanley, P. An Engineer's Approach to the Prediction of Failure Probability of Brittle Components. Proceedings of the British Ceramic Society, 1973; 453-857.

(5) Beierlein, G. Festigkeitsverhalten keramischer Werkstoffe unter mehrachsiger mechanischer Beanspruchung. Dissertation Ingenieurhochschule Zwickau, 1988

(6) Nemeth, N.N. Manderscheid, J.M. y GYeKenYesi, J.P. Ceramics Analysis and Reliability Evaluation of Estructures (CARES). Users and Programmers Manual. NASA Technical Paper 2916, Agosto, 1990.

(7) ThiEmier, Th. Lebensvorhersage für keramische Bauteile unter mehrachsiger Beanspruchung. Diss. Universität. Kalsruhe, 1989.

(8) Batdorf, S.B. y Heinsch, H.L. Jr. J. Am. Ceram. Soc., 61, (7-8), 1978: 355-358.

(9) Shetty, D.K. J. Eng. Gas Turbines Power, 109 (3), 1987; 282-289

(10) AlPA, G. Eng. Frac. Mech. 19, (5), 1984: 881-901.

(11) JAKEL, R. Programm WEIBULL: Ein Subprozessor zur Abschätzung der Sofort- und Zeitausfallwahrscheinlichkeit keramischer Bauteile für das FiniteElemente-Programm MARC. Theorie und Bedienungsanleitung. Handbuch IMW TU Clausthal, Sept. 1994.

(12) JAKEL, R. Ein Betrag zur Berechnung und konstruktiven Gestaltung keramischer Bauteile, angewendet am Beiespiel eines keramischen Heigasventilatorrades. Dissertation zur Erlangung des Grades eines DoktorIngenieurs. IMW TU Clausthal, Julio 1996.

(13) NADLER, P. Beitrag zur Charakterisierung und Berücksichtigung des spezifischen keramischen Festigkeitsverhaltens. Diss. Bergakademie Freiberg, 1989.

(14) Crespo, C. Predicción de vida y optimización de componentes cerámicos. Proyecto fin de carrera (diciembre 1996). 